\title{
QUANTIFICATION AND COMPENSATION OF TEMPORAL DECORRELATION EFFECTS IN POLARIMETRIC SAR INTERFEROMETRY
}

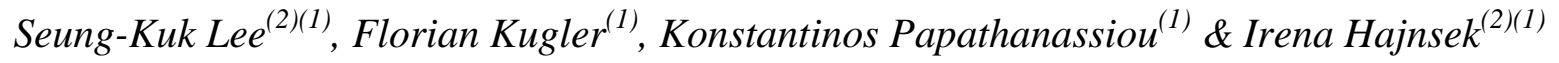 \\ ${ }^{(1)}$ German Aerospace Center (DLR), Microwaves and Radar Institute, Germany \\ ${ }^{(2)}$ ETH Zurich, Institute of Environmental Engineering, Switzerland
}

\begin{abstract}
Temporal decorrelation is a critical issue for a successful Pol-InSAR inversion in case of repeat-pass SAR data, as provided by conventional satellite or airborne SAR systems. This paper proposes estimation and compensation of temporal decorrelation effects by using multi-baseline PolInSAR data. A new approach to quantify different temporal decorrelation levels (one for volume and the other for the ground layer) is performed without resort to the special case of zero spatial baseline interferograms. Both temporal decorrelation coefficients were separately estimated at temporal baselines ranging from 1 to 15 days and compared to height inversion errors caused by them.
\end{abstract}

Index Terms - Pol-InSAR, temporal decorrelation, multi-baseline, forest height inversion

\section{INTRODUCTION}

Several studies showed that Pol-InSAR data especially at lower frequencies such as L- and P-band, are sensitive to the vertical distribution of scatterer along forest height. A model-based inversion of forest height and structural parameters was introduced and demonstrated in [1][2][4][5].

However, in repeat-pass airborne/spaceborne SAR system, temporal decorrelation is the most critical parameter for a successful Pol-InSAR forest parameter inversion. Temporal decorrelation is caused by the dynamic changes and/or the variation of the dielectric properties of the scatterers within the scene occurring in the time between the two SAR acquisitions [5][6]. Temporal decorrelation lowers the estimated coherence and therefore also the estimated volume decorrelation which is directly related to the vertical distribution of scatterers. Biased volume coherence leads to a biased forest parameter estimation in Pol-InSAR inversion [9][10][11]. Due to its random character it is difficult to estimate and compensate temporal decorrelation contribution from the complex interferometric coherence.

In this paper, a quantitative analysis of temporal decorrelation as a function of time using experimental data acquired by DLR's E-SAR system is performed. Then the implementation of a "coherent" multi-baseline inversion technique for compensating temporal decorrelation is evaluated. The behavior and compensation of temporal decorrelation is demonstrated by means of three campaign data sets: BioSAR 2007 [8] and TempoSAR 2008/2009 [10][11].

\section{POL-INSAR INVERSION \& TEMPORAL DECORRELATION}

A widely and successfully used two-layer model, the so-called Random Volume over Ground (RVoG) model [3][4][5] represents coherently two scattering mechanisms: one from the volume and the other from the ground layer. The RVoG model is characterized by having a smaller number of independent physical parameters (assuming no response from the ground in one polarization channel) than observables so that these parameters can be estimated form Pol-InSAR data. However, the model does not account for temporal decorrelation contributions which are a critical factor for forest parameter estimation in case of a conventional repeat-pass SAR system. With a two-layer (volume/ground) scattering model, two different temporal decorrelation processes can be introduced: $\gamma_{T V}$ denotes the correlation coefficient describing the temporal decorrelation of the volume layer and $\gamma_{T G}$ represents the correlation coefficient describing temporal decorrelation of the underlying surface scatterer. Both temporal decorrelation effects can be incorporated in the RVoG model as [6][7]

$$
\tilde{\gamma}(\vec{w})=e^{i \phi_{0}} \frac{\gamma_{T V} \tilde{\gamma}_{V}+\gamma_{T G} m(\vec{w})}{1+m(\vec{w})}
$$

where $\tilde{\gamma}_{V}$ is the complex volume decorrelation and $m$ represents the effective ground-to-volume amplitude ratio accounting for the attenuation through the volume. Equation (1) cannot be solved using a quad-polarization single baseline acquisition due to the additional two unknown parameters $\left(\gamma_{T V}, \gamma_{T G}\right)$. However, even if the general temporal decorrelation scenario of Equation (1) leads to an 
underdetermined problem, special temporal decorrelation scenarios under certain assumptions may be accounted for with multi-baseline acquisitions.

When the temporal baseline is considerably short (i.e. smaller than one hour), it is possible to assume that surface scatterers on the ground are stationary, and dielectric constants are unchanged (i.e. $\gamma_{T G}=1$ ). Thus, the most common temporal decorrelation contributions over forest are mainly wind-induced movement of scatterers only within the volume layer. In the complex plain, the ground point (green rectangular point in Figure 1 (a)) remains unchanged, while the volume coherence $\tilde{\gamma}_{V}$ is shifted towards the origin by the factor $\gamma_{T V}$ as shown in Figure 1 (a). Height errors increase with increasing temporal decorrelation of $\gamma_{T V}$ and the estimation errors are significantly higher for low than for high forest heights [10].

For temporal baselines on the order of days, temporal decorrelation contributions induced by the change of scattering properties on the ground layer cannot be neglected (i.e. $\gamma_{T G}<1$ ). Two temporal decorrelations , $\gamma_{T V}$ and $\gamma_{T G}$ result in a shift of the volume decorrelation $\tilde{\gamma}_{V}$ and the ground point $e^{i \phi_{0}}$ radially towards the origin as shown in Figure 1 (a). Note that the coherence loci decorrelated by both temporal decorrelations determine the biased ground point $e_{p s e u d o}^{i \phi_{0}}$ (blue rectangular point) and cause a ground phase error $\Delta \phi_{0}$ (see Figure 1 (a)). As a consequence of $\Delta \phi_{0}$, the phase center of the volume decorrelation $\tilde{\gamma}_{V}$ is overestimated and leads to a height error in the Pol-InSAR inversion.

Figure 2 shows the ground phase error and the height error induced by different levels of temporal decorrelation on the ground layer $\left(\gamma_{T G}=1.0\right.$ to 0.8 ) as a function of forest height assuming a vertical wavenumber of $\kappa_{z}=0.12 \mathrm{rad} / \mathrm{m}$ and the temporal decorrelation in volume $\gamma_{T V}$ of 0.9. While no ground phase error appears for $\gamma_{T G}=1.0, \Delta \phi_{0}$ increases as $\gamma_{T G}$ decreases and forest height decreases. Figure 2 (b) shows the height error resulting from the phase error shown in Figure 2 (a). Compared to the impact of temporal decorrelation in volume $\gamma_{T V}$ [10], a phase error caused by $\gamma_{T G}$ introduces a smaller bias in the Pol-InSAR inversion.

\subsection{Experimental result for temporal decorrelations}

The TempoSAR campaigns were preformed over the Traunstein test site in 2008 and 2009 to collect L-band PolInSAR data sets with a variety of spatial and temporal baselines. A forest height map obtained from multi-baseline inversion and incoherent combination of the single baseline results was presented in [12]. Using these results (i.e. forest height $h_{v}$ and extinction $\sigma$ ), the volume coherence
$\tilde{\gamma}_{V}\left(h_{v}, \sigma, \kappa_{z}, \theta_{0}\right)$ can be calculated (setting $\phi_{0}=0$ ) and plotted (the green circle in Figure 1 (b)). For any Pol-InSAR acquisitions, the associated volume-only coherence $\tilde{\gamma}\left(\vec{w}_{m=0}\right)$ and the biased ground point $e_{p s e u d o}^{i \phi_{0}}$ are obtained. The ground phase error $\Delta \phi_{0}$ is estimated by the phase difference between $\tilde{\gamma}_{V}\left(h_{v}, \sigma, \kappa_{z}, \theta_{0}\right)$ and $\tilde{\gamma}\left(\vec{w}_{m=0}\right) e_{p s e u d o}^{-\phi_{0}}$. The temporal decorrelation on the ground layer $\gamma_{T G}$ is obtained by the $\mathrm{x}$-intercept of the line defined by $\tilde{\gamma}\left(\vec{w}_{m=0}\right) e_{p s e u d o}^{-\phi_{0}} e^{-\Delta \phi_{0}} \quad$ and $e^{-\Delta \phi_{0}}$ (red circle and blue rectangular points in Figure 1 (b)):

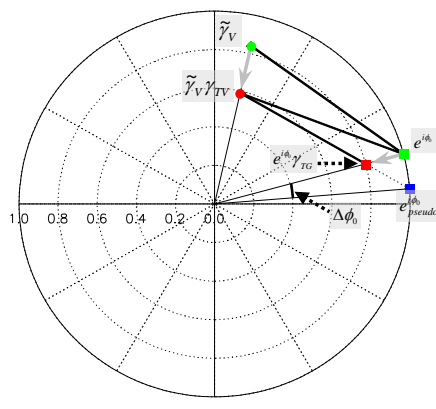

(a)

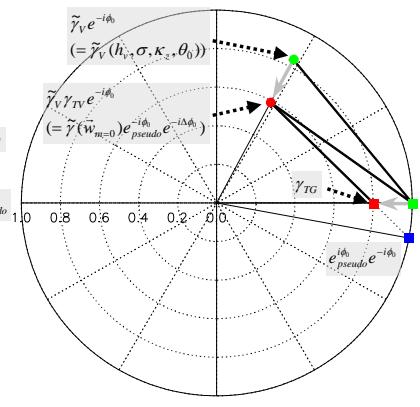

(b)
Figure 1: (a) Coherence loci for the RVoG model with temporal decorrelations of $\gamma_{T V}$ and $\gamma_{T G}$. (b) Coherence loci rotated by the ground phase $e^{-i \phi_{0}}\left(=e_{\text {pseudo }}^{-i \phi_{0}} e^{-i \Delta \phi_{0}}\right)$; Temporal decorrelation on the ground layer $\gamma_{T G}$ is located on the $\mathrm{x}$-axis.

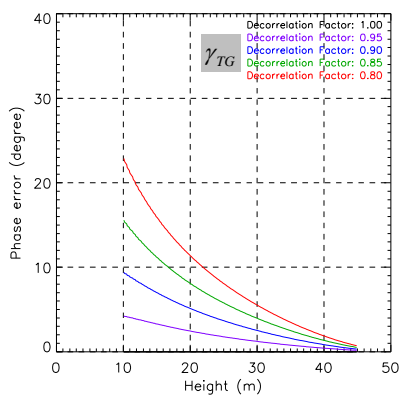

(a)

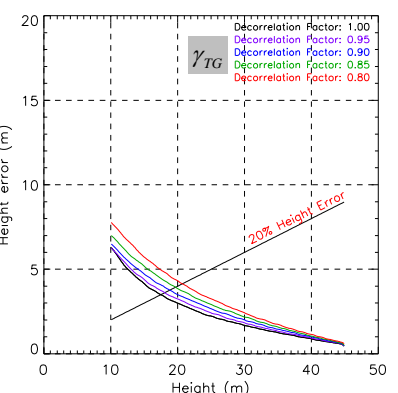

(b)
Figure 2: Errors caused by $\gamma_{T G}$. (a) Ground phase error and (b) height error induced by different levels of temporal decorrelation $\gamma_{T G}$ as a function of forest heights assuming a vertical wavenumber of $\kappa_{z}=0.12 \mathrm{rad} / \mathrm{m}$ and temporal decorrelation of $\gamma_{T V}=0.9$. 


$$
\begin{aligned}
\gamma_{T G}=\operatorname{Re}\left(\tilde{\gamma}\left(\vec{w}_{m=0}\right) e_{p s e u d o}^{-i \phi_{0}} e^{-i \Delta \phi_{0}}\right) \\
\quad-\operatorname{Im}\left(\tilde{\gamma}\left(\vec{w}_{m=0}\right) e_{p s e u d o}^{-i \phi_{0}} e^{-i \Delta \phi_{0}}\right) / \tan \theta_{g r}
\end{aligned}
$$

where $\tan \theta_{g r}$ represents the gradient of the line.

As mentioned, temporal volume decorrelation $\gamma_{T V}$ reduces the amplitude of the volume decorrelation $\tilde{\gamma}_{V}$ in Equation (1). Therefore, $\gamma_{T V}$ can be obtained by the amplitude ratio of $\tilde{\gamma}_{V}\left(h_{v}, \sigma, \kappa_{z}, \theta_{0}\right)$ and $\tilde{\gamma}\left(\vec{w}_{m=0}\right) e_{p s e u d o}^{-\phi_{0}} e^{-\Delta \phi_{0}}$ (green and red circle points in Figure 1 (b)).

The quantitative estimation of $\gamma_{T G}$ and $\gamma_{T V}$ was performed by using Pol-InSAR data sets with temporal baselines up to 15 days. The results are shown in Figure 3. Overall temporal decorrelations of $\gamma_{T V}$ and $\gamma_{T G}$ decreases with an increase of the temporal baseline. However, the decorrelation processes within the volume layer occur much faster than the ones on the ground layer. The reason for this is that the scatterers in the vegetation layer are less stable against wind disturbances than the ones on the ground layer. But, the temporal decorrelations of $\gamma_{T V}$ and $\gamma_{T G}$ depend not only on the wind conditions but also on the rain-induced dielectric changes of volume and ground layer. Looking on the results for one day temporal baseline (Scene_ID: 01xx/02xx, 04xx/05xx and 08xx/09xx) of TempoSAR 2009, temporal decorrelations between $04 \mathrm{xx}$ and $05 \mathrm{xx}$ image pair show a much lower coherence level $\left(\gamma_{T V}=0.60\right.$ and $\gamma_{T G}=$ 0.76) than the others. This may result from changes of dielectric properties caused by the precipitation just before the $05 x x$ acquisition on $12^{\text {th }}$ May 2009.

For the validation of the obtained results, a simulated height error for the estimated $\gamma_{T V}$ and $\gamma_{T G}$ obtained from each temporal baseline for a $\kappa_{z}$ of $0.1 \mathrm{rad} / \mathrm{m}$ and a forest height of $26 \mathrm{~m}$ (mean height value in test site) is calculated and plotted against the real height error of the individual temporal baselines from 1 to 15 days [11]. Figure 4 shows this plot: on the $\mathrm{x}$-axis is the real height error while on the $\mathrm{y}$ axis the simulated height error obtained by using the estimated temporal decorrelations $\left(\gamma_{T V}\right.$ and $\left.\gamma_{T G}\right)$ from Figure 3 are given. The comparison shows a surprisingly high $r^{2}$ of 0.94 with an RMSE of $3.34 \%$. This means that the estimations of $\gamma_{T V}$ and $\gamma_{T G}$ are in accordance with the experimental results achieved.

\section{MULTI-BASELINE POL-INSAR INVERSION}

For the compensation of at least the $\gamma_{T V}$ component a coherent dual baseline inversion is suggested.

In case of multi-baseline Pol-InSAR data, each of the available spatial baselines with its corresponding vertical wave numbers $\kappa_{z i}$ (where $i \in\{1,2\}$ ) provides a set of three different complex coherences $\left[\begin{array}{llll}\tilde{\gamma}\left(\vec{w}_{1}\right) & \tilde{\gamma}\left(\vec{w}_{2}\right) & \tilde{\gamma}\left(\vec{w}_{3}\right)\end{array}\right]^{i}$. One way to compensate for temporal decorrelation $\gamma_{T V}^{i}$ is to estimate first for each single baseline the complex coherence $\tilde{\gamma}\left(\vec{w} \mid \kappa_{z}^{i}\right)$ without ground contribution in the signal $\left(m_{3}=0\right)$. Then, for all baselines the $h_{v}, \sigma$ and $\gamma_{T V}^{i}$ are collected, i.e. the one associated with $\tilde{\gamma}\left(\left\{h_{v}, \sigma, m_{3}=0, \gamma_{T V}^{i}\right\} \mid \kappa_{z}^{i}\right)$. In a second step, $h_{v}, \sigma$ (that are baseline invariant) and $\gamma_{T V}^{i}$ are estimated from

$$
\min _{h_{v}, \sigma, \gamma_{T V}^{i}, \phi_{0}}\left\|\left[\begin{array}{l}
\tilde{\gamma}\left(\vec{w}_{3} \mid \kappa_{z}^{1}\right) \\
\tilde{\gamma}\left(\vec{w}_{3} \mid \kappa_{z}^{2}\right)
\end{array}\right]-\left[\begin{array}{l}
\tilde{\gamma}\left(\kappa_{z}^{1},\left\{h_{v}, \sigma, \gamma_{T V}^{1}\right\}\right) \\
\tilde{\gamma}\left(\kappa_{z}^{2},\left\{h_{v}, \sigma, \gamma_{T V}^{2}\right\}\right)
\end{array}\right]\right\| .
$$

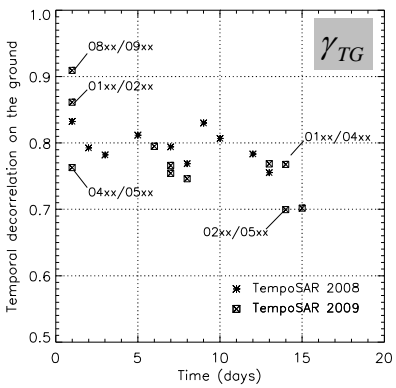

(a)

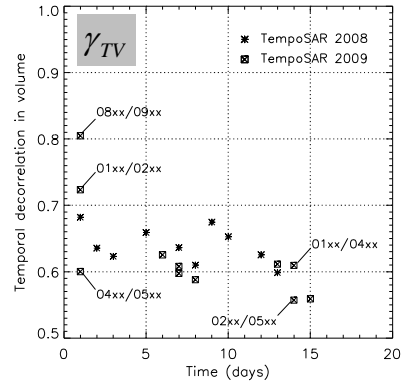

(b)
Figure 3: Mean estimated temporal decorrelations (a) on the ground layer $\gamma_{T G}$ and (b) in volume $\gamma_{T V}$ against temporal baseline of up to 15 days for TempoSAR 2008 and 2009. Asterisk point: TempoSAR 2008 and rectangular point: TempoSAR 2009.

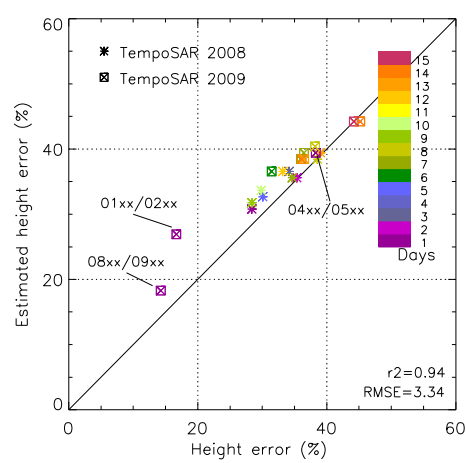

Figure 4: Validation of the estimated height error. X-axis: Height errors obtained directly from Pol-InSAR height inversion with temporal baselines. Y-axis: Height errors estimated by inverting Equation (1) with $\gamma_{T G}$ and $\gamma_{T V}$ from Figure 3 (a) and (b). Color represents the temporal baselines. 


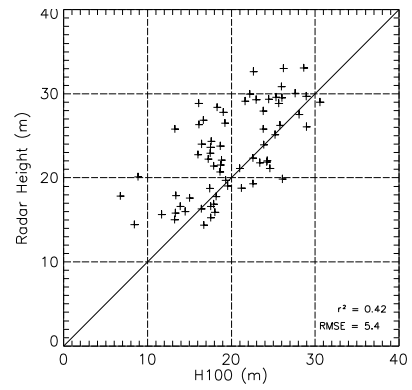

(a)

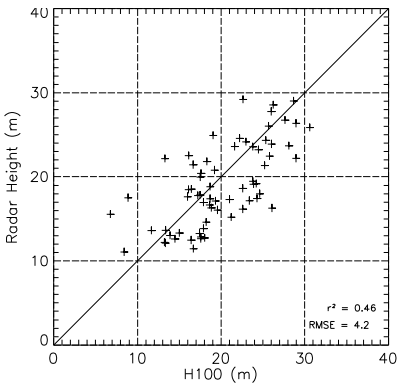

(b)
Figure 5: Estimated forest height vs. lidar derived H100. (a) noncompensation and (b) compensation of temporal decorrelation in the volume layer.

This algorithm is now demonstrated by means of a PolInSAR data set at P-band acquired during the BioSAR 2007 campaign. During the campaign data acquisitions were done on three different dates over the Remningstorp test site: $9^{\text {th }}$ March, $2^{\text {nd }}$ April and $2^{\text {nd }}$ May 2007 [8]. From these three data acquisitions, two temporal baselines on the order of one month could be generated: $9^{\text {th }}$ March $-2^{\text {nd }}$ April (24 days) and $2^{\text {nd }}$ April $-2^{\text {nd }}$ May 2007 (30 days). The Ground component in P-band is dominated by double bounce scattering and has a higher temporal stability than a ground component dominated by surface scattering. Therefore temporal decorrelation of the ground layer at P-band was neglected (i.e. $\gamma_{T G} \cong 1$ in Equation (1)) in the inversion. Dual baseline height inversion was done by means of Equation (3) for the two temporal baselines of the Remningstorp test site and validated against Lidar reference measurements. Figure 5 (a) shows inversion results for the same data set without compensating for temporal decorrelation effects $\left(r^{2}=0.42 \quad \mathrm{RMSE}=5.4 \mathrm{~m}\right)$ with a significant overestimation of forest height. Figure 5 (b) shows the validation plot for the dual-baseline inversion as described by Equation (3) accounting for two different scalar temporal decorrelation coefficients for the volume part $\left(\gamma_{T V}^{1}\right.$ and $\left.\gamma_{T V}^{2}\right)$. The inversion performance is significantly improved $\left(r^{2}=0.46\right.$ RMSE $\left.=4.2 \mathrm{~m}\right)$ and the height bias is removed.

\section{CONCLUSIONS}

In this paper, Pol-InSAR temporal decorrelation model with two different temporal decorrelation coefficients was discussed. The estimation of different temporal decorrelation in time was quantitatively achieved. In order to overcome the impact of temporal decorrelation in the volume layer a "coherent" dual-baseline Pol-InSAR inversion was suggested. First results showed that a compensation of two scalar temporal decorrelation factors (one for each baseline) is feasible.

\section{REFERENCES}

[1] S. R. Cloude and K. P. Papathanassiou 1998, "Polarimetric SAR Interferometry," IEEE Transactions on Geoscience and Remote Sensing, vol. 36, no. 5, pp. 1551-1565, September 1998.

[2] K. P. Papathanassiou and S. R. Cloude, "Single baseline Polarimetric SAR Interferometry," IEEE Transactions on Geoscience and Remote Sensing, vol. 39, no. 11, pp. 23522363, 2001.

[3] R. N. Treuhaft, S. N. Madsen, M. Moghaddam, and J. J. van Zyl, "Vegetation characteristics and underlying topography from interferometric radar," Radio Sci., vol. 31, no. 6, pp. 1449-1495, 1996.

[4] S. R. Cloude and K. P., Papathanassiou, "Three-stage inversion process for polarimetric SAR interferometry," IEE Proceedings - Radar Sonar and Navigation, vol. 150, no. 3, pp. 125-134, 2003.

[5] I. Hajnsek, F. Kugler, S.-K. Lee, K. P. Papathanassiou, "Tropical-Forest-Parameter Estimation by means of PolInSAR: The INDREX II Campaign," IEEE Transactions on Geoscience and Remote Sensing, vol. 47, no. 2, pp. 481 - 493, February 2009.

[6] K. P. Papathanassiou and S. R. Cloude, "The Effect of Temporal Decorrelation on the Inversion of Forest Parameters from Pol-InSAR Data," Proceedings IGARSS'03 (CD-ROM), Toulouse, France, 2003.

[7] M. Lavalle, M. Simard and S. Hensley, "A Temporal Decorrelation Model for Polarimetric Radar Interferometers," IEEE Transactions on Geoscience and Remote Sensing, (accepted to publication).

[8] S. K. Lee, F. Kugler, I. Hajnsek and K. P. Papathanassiou, "Quantifying Temporal Decorrelation over Boreal Forest at Land P-band", Proceedings of European Conference on Synthetic Aperture Radar, EUSAR'08, Friedrichshafen, Germany, 2008.

[9] S. K. Lee, F. Kugler, I. Hajnsek and K. P. Papathanassiou, "The Impact of Temporal Decorrelation over Forest Terrain in Polarimetric SAR Interferometry," POLinSAR, ESA-ESRIN, Frascati, Italy, January 2009.

[10] S. K. Lee, K. P. Papathanassiou and A. Moreira. "Forest Height Estimation by means of Pol-InSAR Limitations posed by Temporal Decorrelation," ALOS Kyoto \& Carbon Initiative Science Report - Phase 1, JAXA TKSC, Tsukuba, Japan, 2009.

[11] S. K. Lee, F. Kugler, I. Hajnsek and K. P. Papathanassiou, "The Impact of Temporal Decorrelation over Forest Terrain in Polarimetric SAR Interferometry," POLinSAR, ESA-ESRIN, Frascati, Italy, January 2009.

[12] S. K. Lee, F. Kugler, I. Hajnsek and K. P. Papathanassiou, "Multibaseline Polarimetric SAR interferometry Forest Height Inversion Approaches," POLinSAR, ESA-ESRIN, Frascati, Italy, January 2011. 